\title{
IDENTIFICAÇÃO PARAMÉTRICA DO SISTEMA COMPOSTO PELO COMPLEXO PERNA-PÉ COM REDES NEURAIS ARTIFICIAIS
}

\author{
Erica R. M. D. MAChado ${ }^{1}$, Feliphe A. M. dos $\operatorname{SAnTOS}^{2}$, MARCElo C. M. TeIXeIRA ${ }^{2}$, Aparecido A.DE

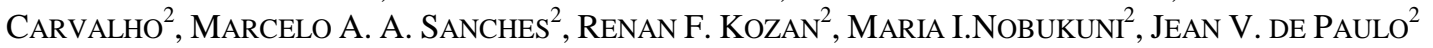 \\ 1. Laboratório de Tecnologia Assistiva, Depto. de Matemática, FEIS-UNESP \\ Caixa Postal 31, 15385-000 Ilha Solteira, SP \\ E-mails: daruichi@mat.feis.unesp.br
}

2. Laboratório de Instrumentação, Departamento de Engenharia Elétrica, FEIS-unesp

E-mails: Isi@engineer.com

\begin{abstract}
This work proposes the use of Hopfield Neural Networks in parametric identification of complex system comprised of the leg and foot. This research is part of a multidisciplinary project that integrates the control systems and instrumentation to control the movements of the lower limbs in paraplegic patients. The objective is to identify the plant parameters and perform simulations of the system, before the application of the control laws in the patient.
\end{abstract}

Keywords_-System Identification, Artificial intelligence techniques to control, Movements of the lower limbs, Artificial Neural Networks

\begin{abstract}
Resumo- Este trabalho propõe o uso de Redes Neurais de Hopfield na identificação paramétrica do sistema complexo composto da perna e do pé. Esta pesquisa é parte de um projeto multidisciplinar que integra os sistemas de controle e instrumentação para controlar os movimentos dos membros inferiores de pacientes paraplégicos. O objectivo é o de identificar os parâmetros de planta e realizar simulações do sistema, antes da aplicação das leis de controle no paciente.
\end{abstract}

Palavras-chave— Identificação de Sistemas, Técnicas de inteligência artificial em controle, Movimentos de membros inferiores, Redes Neurais Artificiais.

\section{Introdução}

Anualmente, centenas de pessoas são acometidas por algum tipo de lesão medular no Brasil e no mundo, que comprometem suas capacidades motoras especialmente em membros inferiores.

Cabe destacar que, após a lesão medular, os músculos atrofiam rapidamente, principalmente os músculos grandes da coxa. Uma das consequências da atrofia muscular é que as atividades do coração e do pulmão são reduzidas, fazendo com que as condições de saúde sejam deterioradas.

Quando um indivíduo não exercita ou movimenta o membro acometido por uma lesão, pode ter seu estado clínico comprometido, influenciando diretamente na qualidade de vida do mesmo.

A Estimulação Elétrica Neuromuscular Funcional (EENF) pode auxiliar o sistema circulatório, aumentando a circulação de sangue no membro paralisado, dentre outros benefícios (CRAMERI et al., 2004). A EENF, em combinação com sensores adequados e técnicas de controle, pode ser explorada para produzir movimentos funcionais, podendo ajudar a restaurar algumas funções motoras e, aplicada em níveis adequados, pode ser tão eficaz no fortalecimento muscular quanto uma contração muscular voluntária.

Um grande desafio que se enfrenta quando se aplica EENF nos membros inferiores paralisados é evitar a hiperestimulação e adiar a fadiga muscular, tanto quanto possível.
Quando se trabalha com sistema em malha fechada, pode-se controlar de maneira mais eficiente a estimulação elétrica, propiciando um melhor controle dos movimentos e evitando uma fadiga mais rápida dos músculos envolvidos no processo. E para iniciar as rotinas de acompanhamento do progresso do tratamento, deve-se obter um controlador que, após a eletroestimulação, mantenha a posição da perna em um ângulo desejado.

Entretanto, antes de se iniciar o projeto do controlador, os parâmetros que compõe as equações dinâmicas do sistema devem ser obtidos e estes variam com o estado de saúde e emocional do paciente.

A identificação dos parâmetros de sistemas dinâmicos parcialmente desconhecidos é um assunto de grande importância prática, pois uma vez conhecido o modelo matemático, podem-se utilizar resultados clássicos para projetar um controlador de modo que o sistema controlado apresente um desempenho desejado especificado previamente.

A importância deste processo é que, através dos parâmetros da planta, se consegue projetar, posteriormente, o conjunto controlador-estimulador, observando-se a resposta temporal através de simulações, evitando-se que os primeiros testes reais de controle sejam aplicados em pacientes.

Os parâmetros da planta do complexo perna-pé são altamente variáveis (Ferrarim e Pedotti, 2000) e podem mudar durante uma sessão de tratamento. Assim, é necessária a utilização de um algoritmo que realize uma identificação rápida dos parâmetros, com poucas amostras. Este ajuste permite que a carga do 
eletro-estimulador seja adequada entre as aplicações, diminuindo assim, a fadiga muscular.

Entretanto, na obtenção dos parâmetros com os algoritmos clássicos de identificação ocorre um atraso devido ás condições iniciais da planta, pois, no início da eletro-estimulação o sistema encontra-se em um estado inercial e o ângulo de repouso não é nulo.

Em Teixeira et al., (1996) foi proposto um algoritmo de identificação paramétrica com as Redes Neurais de Hopfield (Hopfield, 1982), que identifica os parâmetros eliminando os efeitos das condições iniciais da planta. Este algoritmo é adequado para o problema, pois, todas as informações obtidas pelo sistema de aquisição de dados são utilizadas e não há atrasos na identificação dos parâmetros.

\section{Propósito}

O objetivo deste trabalho é a identificação paramétrica do sistema composto pelo complexo perna-pé utilizando o algoritmo de identificação com Redes Neurais de Hopfield apresentado em Teixeira et al., (1996).

\section{Métodos}

Inicialmente será apresentado um breve resumo sobre Redes Neurais de Hopfield e o algoritmo de identificação proposto em Teixeira et al., (1996). Em seguida será apresentado o modelo biológico proposto por Ferrarin e Pedotti (2000) e, por fim, a implementação computacional do algoritmo no software MATLAB/SIMULINK.

\subsection{Rede Neural de Hopfield}

A Rede Neural Artificial de Hopfield (Hopfield, 1982) é uma rede auto-associativa no qual os valores sinápticos dos neurônios são atualizados localmente. O estado do neurônio depende apenas de sua conexão sináptica, ou de seu peso, para um dado instante e, portanto, não estabelece nenhum procedimento de treinamento para determinar a matriz de pesos, como ocorre na maioria das outras redes. Um procedimento de projeto baseado na função de Lyapunov é usado para determinar a matriz peso.

Hopfield projetou uma matriz peso $T$ e o vetor de entrada $I$ de modo a formar uma função denominada de função energia $E$, que pode ser minimizada. Muitos problemas podem ser representados por uma função quadrática e serem convertidos a uma função energia $E$. Como a rede de Hopfield é capaz de minimizar $E$, ela também pode minimizar a função original. O problema se resume em obter uma função energia adequada.

O sistema de identificação utilizando a rede de Hopfield é um sistema de identificação paramétrica, no qual os parâmetros são estimados pela minimiza- ção de uma função energia. O estado de mínimo da rede determina os parâmetros do sistema.

O modelo de Hopfield (1982) consiste de várias unidades de processamento, chamadas "neurônios", interligadas. Um neurônio "i" é modelado por:

$$
\frac{d U_{i}}{d t}=S_{i} \frac{U_{i}}{R_{i}}+I_{i}, V_{i}=\alpha_{i} g\left(\beta_{i} U_{i}\right), U_{i} \text { e } V_{i} \in R
$$

onde $S_{i}$ é a entrada, $U_{i}$ o estado, $V_{i}$ a saída, $\alpha_{i}, \beta_{i}$ e $R_{i}$ são constantes positivas, $I_{\mathrm{i}}$ é uma constante e $\mathrm{h}($. ) é uma função sigmóide, sendo que normalmente, $\mathrm{h}\left(U_{i}\right)=\tanh \left(U_{i}\right)$.

Hopfield estudou a possibilidade da determinação de um mínimo local da função energia

$$
E_{h}=-\frac{1}{2} \sum_{i=1}^{n} \sum_{j=1}^{n} T_{i j} V_{i} V_{j}-\sum_{i=1}^{n} I_{i} V_{i}+\sum_{i=1}^{n} \frac{1}{R_{i} \alpha_{i}} \int_{0}^{\frac{V_{i}}{\alpha_{i}}} h^{-1}(v) d V,
$$

sendo $\alpha_{i}, I_{i}, T_{i j}=T_{j i}$, e $j=1, \ldots, n$ constantes e $h^{-1}(v)$ é a função inversa de $h(v)$, utilizando um sistema dinâmico composto de neurônios interligados. Hopfield demonstrou que o sistema dinâmico (3)-(4)

$$
\begin{gathered}
d U_{i} / d t=\sum_{j=1}^{N} T_{i j} V_{j}-U_{i} / R_{i}+I_{i}, i=1, \ldots, n, \\
V_{i}=\alpha_{i} g\left(\beta_{i} U_{i}\right),
\end{gathered}
$$

converge, quando $\mathrm{t} \rightarrow \infty$, para um ponto de equilíbrio $d U_{i} / d \in 0, i=1, \ldots n$, que é um mínimo local de $E$ em (2).

Uma forma válida, simples e muito utilizada dos resultados de Hopfield é quando $\mathrm{R}_{\mathrm{i}} \rightarrow \infty, \mathrm{i}=1, \ldots, \mathrm{n}$ em (2) e (3), o que resulta na remoção do último termo à direita de $E$ em (2) e do termo $U_{i} / R_{i}$ em (3).

\subsection{Algoritmo de Identificação proposto em Teixeira et al., (1996)}

Seja a planta linear e invariante no tempo dada por:

$$
\dot{x}=A_{p} x+B_{p} u
$$

onde $x \in \mathfrak{R}^{n}$ e $u \in \mathfrak{R}$.

Em Teixeira et al., (1996) foi proposta uma configuração que permite a identificação de sistemas dinâmicos lineares e invariantes no tempo dado por (5), sem a disponibilidade de $\dot{\mathrm{x}}$ e ainda independente das condições iniciais da planta $x(0)$ e o seu efeito no transitório dos sinais filtrados.

Considerando $G(s)$ como a função de transferência dos filtros de estado, sendo que $G(s)$ possui o número de pólos maior do que o número de zeros e é estável, definiu-se para a planta (5) os sinais x e u filtrados, denominados $x_{f}$ e $u_{f}$, respectivamente.

$$
X_{f}(s)=G(s) X(s) \text { e } U_{f}(s)=G(s) U(s) .
$$

Para a obtenção do resultado desejado, o filtro $G(s)$ foi aplicado em ambos os membros de (5). Definindo-se $g(t)$ como a transformada inversa de $G(s)$, tem-se então, utilizando-se a integral de convolução e (6), que: 


$$
\begin{aligned}
\int_{o}^{t} g(t-\tau) \dot{x}(\tau) d \tau & =A_{p} \int_{o}^{t} g(t-\tau) x(\tau) d \tau \\
& +B_{p} \int_{o}^{t} g(t-\tau) u(\tau) d \tau \\
\int_{0}^{t} g(t-\tau) \dot{x}(\tau) d \tau & =A_{p} x_{f}(t)+B_{p} u_{f}(t)
\end{aligned}
$$

Considere agora um filtro de estado de $1^{\underline{0}}$ ordem dado por $G(s)=a /(s+a), a>0$. Então (8) pode ser colocada na seguinte forma:

$$
A_{p} x_{f}+B u_{f}+a e^{-a t} x(0)-a x(t)+a x_{f}(t)=0
$$

Vide (Teixeira et al., 1996).

Definindo $A$ e $B$ como os valores estimados de $A_{p}$ e $B_{p}$ da planta, respectivamente, então se pode usar o erro

$$
\Delta e_{q}=A x_{f}+B u_{f}+a e^{-a t} x(0)-a x(t)+a x_{f}(t)
$$

e a função energia

$$
E=1 / T \int_{0}^{T} \Delta e_{q}(t)^{T} \Delta e_{q}(t)
$$

para a estimação de $A_{p}$ e $B_{p}$ utilizando os resultados de Hopfield (1982).

Para efetuar as simulações, foi definida uma função energia que corresponde a uma discretização da apresentada em (11).

$$
E_{p}=\sum_{j=1}^{M} \frac{1}{2} \Delta e_{q}(j T)^{T} \Delta e_{q}(j T)
$$

onde $\Delta e_{q}(t)$ foi definido em (10).

Desenvolvendo-se (12), pode-se colocá-la na forma abaixo, desprezando-se o termo constante:

$$
E_{P H}=V^{T} T V+I^{T} V .
$$

Para efeito de ilustração, considere um sistema de $2^{-}$ordem, $n=2$ em (5),

$$
\begin{gathered}
T=-\sum_{j=0}^{T}[M] \\
M=\left[\begin{array}{cccccc}
x_{1}{ }^{j} x_{1}{ }^{j} x_{2}{ }^{j} & 0 & 0 & x_{1} u^{j} & x_{2}{ }^{j} u^{j} \\
x_{1} x_{x_{2}}^{j} & x_{2}{ }^{j^{2}} & 0 & 0 & x_{2}{ }^{j} u^{j} & 0 \\
0 & 0 & x_{1}{ }^{2} & x_{1}{ }^{j} x_{2}{ }^{j} & 0 & x_{1}{ }^{j} u^{j} \\
0 & 0 & x_{1}{ }^{j} x_{2}{ }^{j} & x_{2}{ }^{j} & 0 & x_{2}{ }^{j} u^{j} \\
x_{1}{ }^{j} u^{j} & x_{2}{ }^{j} u^{j} & 0 & 0 & u^{j^{2}} & 0 \\
x_{2}{ }^{j} u^{j} & 0 & x_{1}{ }^{j} u^{j} & x_{2}{ }^{j} u^{j} & 0 & u^{j^{2}}
\end{array}\right]
\end{gathered}
$$

sendo $\quad x_{i}^{j}=\int_{0}^{j T} g(t-\tau) x_{i}(\tau) d \tau$,

$$
\begin{aligned}
& V^{T}=\left[a_{11} \ldots a_{1 n} \ldots a_{n 1} \ldots a_{n n} b_{1} \ldots b_{n}\right], \\
& I^{T}=\sum_{j=1}^{M}\left[x_{1}\left(x_{1}(j \Delta)-x_{1}(0)\right)\right. \\
& x_{2}\left(x_{1}(j \Delta)-x_{1}(0)\right) \\
& x_{2}\left(x_{2}(j \Delta)-x_{2}(0)\right) \\
& u\left(x_{1}(j \Delta)-x_{1}(0)\right) \\
& \left.u\left(x_{2}(j \Delta)-x_{2}(0)\right)\right]
\end{aligned}
$$

Assim, os elementos de A e B da planta podem ser estimados por uma Rede Neural de Hopfield dada em (3)-(4):

$$
\dot{U}_{i}=T V+I
$$

$$
V_{i}=\alpha_{i} h\left(\beta_{i} U_{i}\right)
$$

e se a entrada for persistentemente excitante, então teremos um único mínimo local e global e a estimação ocorrerá sem problemas.

$\mathrm{O}$ erro $\Delta e_{\mathrm{q}}$ em (10) considera a influência da condição inicial $\mathrm{x}(0)$ com a dinâmica do filtro e assim, a estimação fica independente deste transitório. A proposta é obter uma identificação rápida, sem desprezar nenhuma informação fornecida pelo sistema de aquisição de dados.

\subsection{Modelo Matemático de Ferrarin e Pedotti (2000)}

O modelo proposto por Ferrarin e Pedotti (2000) relaciona a largura do pulso aplicado com o torque gerado na articulação do joelho.

$\mathrm{Na}$ modelagem os autores consideram o membro inferior como uma cadeia cinemática aberta composta de dois segmentos rígidos: a coxa e o complexo perna-pé, conforme ilustra a Figura 1.

A eletroestimulação funcional é aplicada ao músculo quadríceps, por meio de eletrodos fixados na pele da coxa do paciente. O objetivo é provocar a contração do músculo, visando posicionar o ângulo do joelho em um valor especificado.

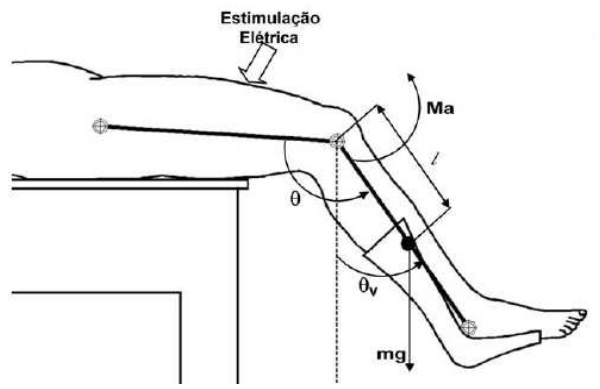

Figura 1: Conjunto Perna-Pé Proposto por Ferrarin e Pedotti.

- $\theta$ : ângulo do joelho (ângulo entre coxa e canela no plano sagital);

- $l$ : é a distância entre o joelho e o centro da massa do complexo canela-pé;

- $\theta_{v}$ : é o ângulo da canela (ângulo entre a canela e o sentido vertical no plano sagital);

- $m$ : massa do complexo canela - pé;

- $g$ : aceleração da gravidade;

- $M_{a}$ : torque ativo do joelho produzido por estimulo elétrico.

Os movimentos da junção do tornozelo não foram considerados, reduzindo o número de graus de liberdade, evitando toda a modificação das características passivas do joelho provocado pelo movimento do tornozelo.

O equilíbrio dinâmico destes componentes, em torno da junção do joelho, pode ser expresso pela seguinte equação diferencial não linear de segundaordem:

$$
J \ddot{\theta}_{v}=-m g l \operatorname{sen}\left(\theta_{v}\right)+M_{s}-B \dot{\theta}+M_{a}
$$

- J é o momento inercial do complexo de canela-pé; 
- $\dot{\theta}$ é a velocidade angular comum do joelho;

- $\ddot{\theta}_{v}$ é a aceleração angular da canela;

- $B$ é o coeficiente de atrito viscoso;

- $M_{S}$ é o torque devido ao componente de rigidez;

A função de transferência para o modelo foi obtida em Ferrarin e Pedotti, (2000) e é dada por:

$$
H(s)=\frac{G /(J \tau)}{s^{3}+\frac{(J+B \tau)}{J \tau} s^{2}+\frac{(B+k \tau)}{J \tau} s+\frac{k}{J \tau}}
$$

A equação de movimento foi linearizada em torno de um ponto de operação e em seguida foram obtidas as equações de estados. A equação (22) apresenta a equação de estados da função de transferência (21). Vide (Teixeira et al., 2007).

$$
\left[\begin{array}{c}
\dot{x}_{1} \\
\dot{x}_{2} \\
\dot{x}_{3}
\end{array}\right]=\left[\begin{array}{ccc}
0 & 1 & 0 \\
0 & 0 & 1 \\
\frac{-k}{J \tau} & \frac{-(B+k \tau)}{J \tau} & \frac{-(J+B \tau)}{J \tau}
\end{array}\right]\left[\begin{array}{l}
x_{1} \\
x_{2} \\
x_{3}
\end{array}\right]+\left[\begin{array}{l}
0 \\
0 \\
1
\end{array}\right] \Delta P
$$

Ferrarin e Pedotti (2000) forneceram uma tabela com valores de $\tau$ para entrada tipo degrau e rampa para diversos pacientes sadios e hemiplégicos e um gráfico com valores do ganho estático $\mathrm{G}$ da função de transferência identificada, para cada paciente e para várias frequências.

Considerando um dos pacientes hemiplégicos foram fornecidos os seguintes valores: $G=42500 \mathrm{Nm} / \mathrm{s}$, $J=0.362\left[\mathrm{kgm}^{2}\right], \quad m=4.37[\mathrm{~kg}], \quad B=0.27[$ N.m.s $/ \mathrm{rad}]$, $\mathrm{E}=2.024[1 / \mathrm{rad}], \quad \omega=2.918[\mathrm{rad}], \quad l=23.8[\mathrm{~cm}]$, $\lambda=41.208[\mathrm{~N} . \mathrm{m} / \mathrm{rad}]$.

A equação (22) pode ser representada por:

$$
\left[\begin{array}{l}
\dot{x}_{1} \\
\dot{x}_{2} \\
\dot{x}_{3}
\end{array}\right]=\left[\begin{array}{ccc}
0 & 1 & 0 \\
0 & 0 & 1 \\
a_{31} & a_{32} & a_{33}
\end{array}\right]\left[\begin{array}{l}
x_{1} \\
x_{2} \\
x_{3}
\end{array}\right]+\left[\begin{array}{l}
0 \\
0 \\
1
\end{array}\right] \Delta P
$$

sendo $\mathrm{a}_{31}=-37.8359, \mathrm{a}_{32}=-36.6967, \mathrm{a}_{33}=-1.7403$.

$\mathrm{O}$ algoritmo de identificação foi aplicado neste sistema linearizado e as simulações foram realizadas no software MATLAB/SIMULINK e foram obtidos resultados para condições não nulas da planta.

\section{Resultados}

As simulações foram realizadas considerando o período de integração $\mathrm{Ts}=0.01 \mathrm{~s}$ as seguintes condições iniciais nulas dos filtros de estados e condições da planta $\left[x_{1}(0) \quad x_{2}(0) \quad x_{3}(0)\right]=\left[\begin{array}{lll}3 & 3 & 3\end{array}\right]$.

A Figura 2 apresenta os resultados das simulações em relação ao número de amostras (NA). Os parâmetros foram identificados com apenas 5 amostras.

\section{Discussões e Conclusões}

Este trabalhou apresentou resultados de simulação para a identificação paramétrica de um sistema biológico.

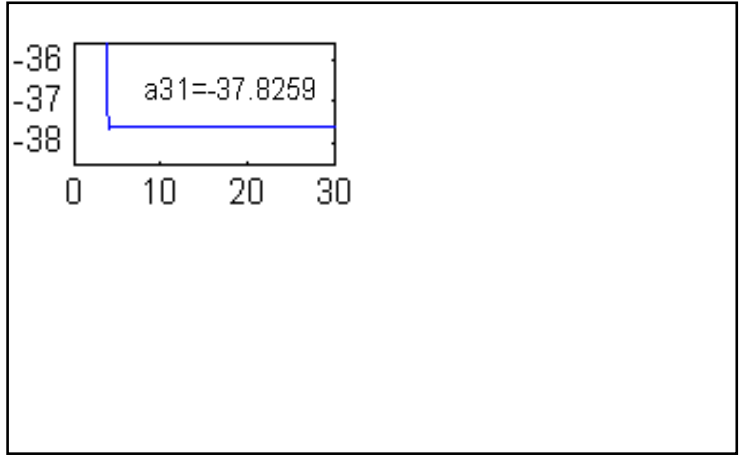

Figura 2: Parâmetros Identificados.

As simulações mostraram que os parâmetros podem ser obtidos com poucas amostras. Estes resultados são importantes para sessões de EENF porque os parâmetros da planta são afetados pela fadiga muscular e várias identificações devem ser realizadas em uma mesma sessão. A identificação rápida dos parâmetros permite que as leis controle sejam ajustadas melhorando na qualidade do tratamento.

\section{Agradecimentos}

Os autores desejam agradecer à Fundação de Amparo à Pesquisa do Estado de São Paulo- FAPESP pelo apoio recebido.

\section{Referências Bibliográficas}

Crameri R., M., Cooper, P., Sinclair P.J., Bryant, G, Weston, A. (2004). Effect of load during electrical stimulation training in spinal cord injury. Muscle \& Nerve, 29:01, p. 104-111. DOI: 10.1002/mus. 10522

Ferrarin, M.; Pedotti, A. (2000). The Relationship Between Electrical Stimulus and Joint Torque: A Dynamic Model, IEEE Trans. Rehabil. Eng. v.1. 8, n.3, p. 342-352, 2000.

Hopfield, J. J. Neural Networks and Physical Systems with Emergent Collective Computational Abilities, (1982) Proc. Natl. Acad. Sci. v.79, p.2554-2558. DOI: 10.1073/pnas.79.8.2554

Teixeira, M. C. M.; Deacto, G. S.,Gaino,R.; Assunção, E.; Carvalho, A. A; Machado, E. R. D.; da Silva, T. I. (2007). Projeto de um Controlador Linear para Variar o Ângulo de Articulação do Joelho de um Paciente Paraplégico. In: 6th Brazilian Conference on Dynamics, Control and Their Applications, São José do Rio Preto-SP, p. 950-956.

Teixeira, M. C. M.; Machado, E. R. M. D.; Assunção, E. Identificação Rápida de Sistemas Dinâmicos Utilizando Redes Neurais de Hopfield. In: Congresso Brasileiro de Automática, 1996, São Paulo. Anais do XI Congresso Brasileiro de Automática, v.1. p. 543548, 1996. 